\title{
COMPARATIVE ANALYSIS OF TECHNOLOGY ADVANCEMENT FROM SINGLE GATE TO MULTI-GATE MOSFET
}

\author{
Abhishek Kumar ${ }^{1}$, Anil Kumar Swain ${ }^{2}$ \\ ${ }^{1} M-T E C H$ Student ,School of Electronics Engineering, KIIT University, Odisha, India. \\ ${ }^{2}$ M-TECH Student ,School of Electronics Engineering, KIIT University, Odisha, India.
}

\begin{abstract}
Among the entire contender in modern microelectronics,DG-MOSFET is a front line runner in planar technology. Itsunique structure allows scaling the device at sub-nanometer region and mimicking the electrical characteristics of a MOSFET.Here simulation of NMOS, SOI-NMOS, and DG-NMOS is presentedand relative comparison among short channel characteristics ispresented.It has been seen that among all the above stated device, DG-MOSFET possess better immune to leakage current with betterDIBL, whereas SOI MOSFET have better driving capacity.
\end{abstract}

KeyWords:SOI-MOSFET, DG-MOSFET, UTB, DIBL,SCES

\section{INTRODUCTION}

In this modern world of technology, where unlimited functions need to be done using limited resources so a lot ofresources and work force is devoted to miniaturization of a transistor so-called "Scaling" [1] Scaling affects the density, speed, functionality, power dissi-pation and cost of an IC [2,3] According to Moore's law transistor is considered as a fundamental element of a digital IC. Now a days scaling reachedpractical limits where it cannot retains the original characteristic due to the presence of short channel effects(SCEs).Due to SCEs devices power dissipation increases. The SCEscomes into picture in the presence if high electric field.This electric field lines are present between drain and source regions. As the device size reduced drain get closer tothe source and punch through effect is observed [4].Here electrostatic potential for NMOS, SOI-MOSFET, and DG-MOSFET for lightly doped channel is obtained. The three structure are compared for subthreshold swing, Ion, Ioff,DIBL and channel potential. The paper is organized as follows: Section 2 discuss the device structures. Section 3 simulation of short channel characteristics of planar device such as NMOS, SOI-NMOS, DG-NMOS. The simulation of result and comparison of different device based on performance parameter havebeen given in Section 4 and finally Section 5 concludes thepaper. Paragraph comes content here. Paragraph comes content here. Paragraph comes content here.

\section{DEVICE STRUCTURE}

The schematic structure of NMOS, SOI-MOSFET, DGMOSFET is shown in figure 1 . In all structure gate length $(\mathrm{Lg})$

\section{$* * *$}

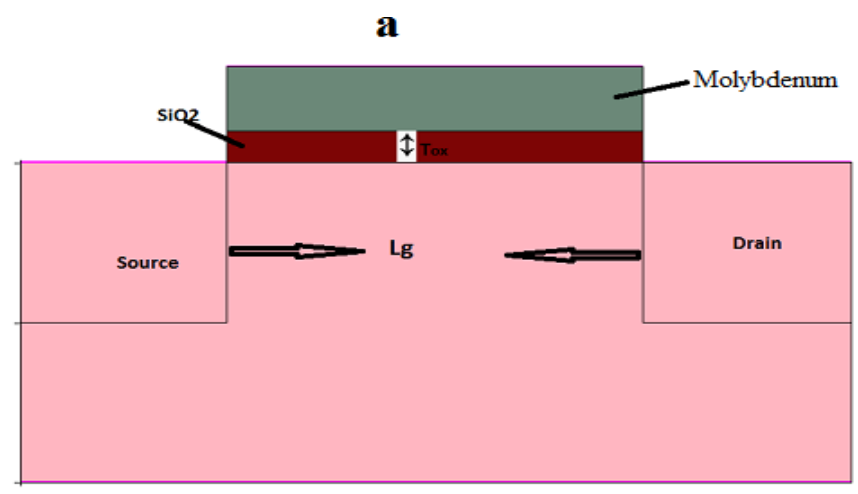

b

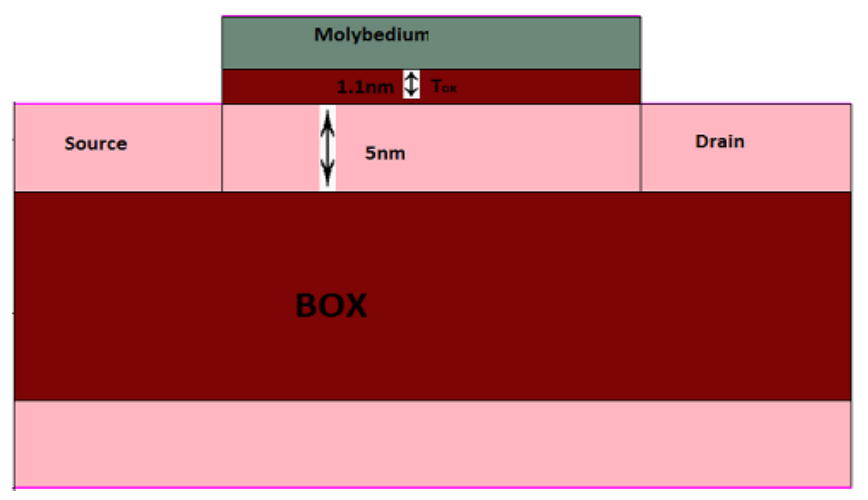

c

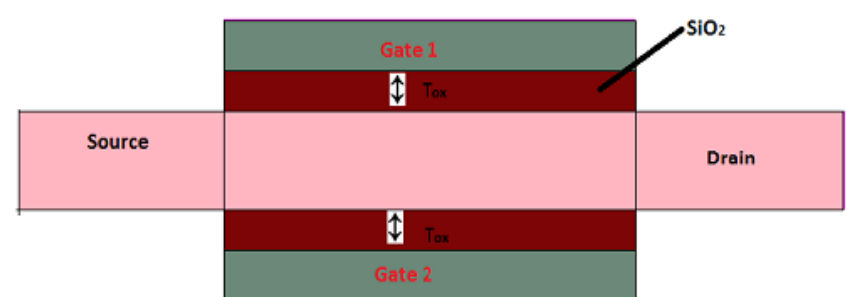

Fig. 1. Schematic structure of a. NMOS b. SOI-MOSFET c. DG-MOSFET, Respectively. 
Table-I. Device Dimension and Doping Concentration

\begin{tabular}{|l|l|}
\hline Oxide Thickness (Tox) & $1.1 \mathrm{~nm}$ \\
\hline Gate Length (Lg) & $22 \mathrm{~nm}$ \\
\hline Source/Drain Doping(ND) & $5 \times 10^{18}$ \\
\hline Channel Doping (NA) & $1 \times 10^{15}$ \\
\hline
\end{tabular}

is fixed as $22 \mathrm{~nm}$. Width of channel is taken as $5 \mathrm{~nm}$ in SOIMOSFET and DG-MOSFET. Total width of device is $10 \mathrm{~nm}$. The Oxide thickness Tox $=1.1 \mathrm{~nm}$ (EOT) is taken. Molybdenumis used gate material.

\section{SHORT CHANNEL CHARACTERISTICS}

\subsection{Sub-Threshold Characteristic}

It is assumed that $\mathrm{Id}=0$ for $\mathrm{Vgs}<\mathrm{Vth}$ but there is finite Current flow called Ioff current or sub threshold current. Id Decreases exponentially below Vth.It is one the main cause of static power dissipation.Fig. 2 shows Ion and Ioff of a different MOSFET. Ioncurrent is maximum in SOI MOSFET indicates maximumdriving capacity but it has also largest Ioff (sub thresholdcurrent). On the other, hand least sub threshold leakage currentfound in DG-MOSFET.

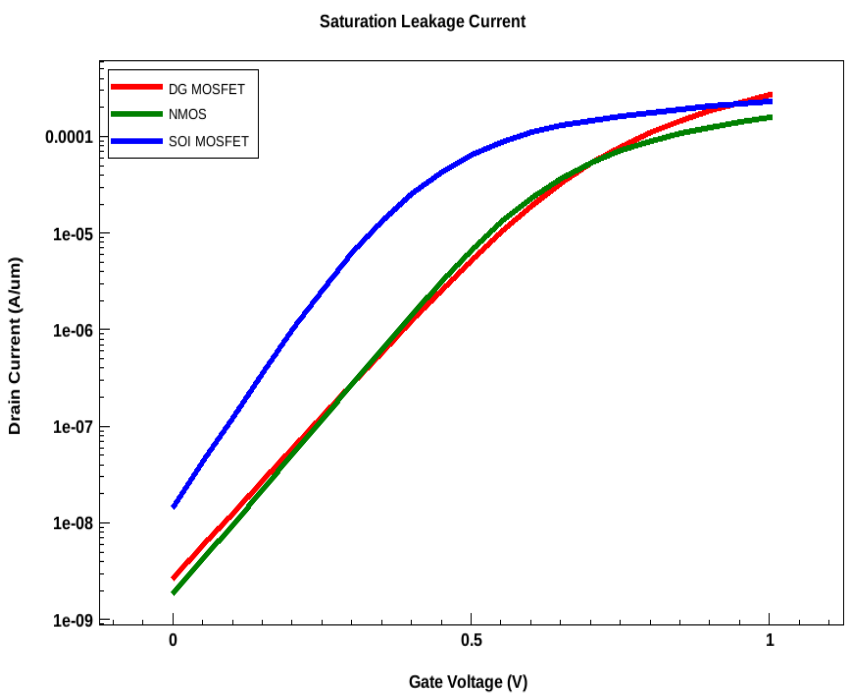

Fig. 2. Ion and Ioff current

\subsection{Sub-Threshold Swing}

It is change in gate voltage that must be applied in order to create one-decade increase in the output current. More sharp the slope, more quickly the device moves from $\mathrm{ON}$ to $\mathrm{OFF}$ state

Sub-threshold Swing $=\frac{d V g s}{\operatorname{dlog} I d s}$

Fig. 3 shows sub-threshold swing of DG-MOSFET is greaterthan SOI-MOSFET but slightly greater than NMOS, so SOI-MOSFET takes more time to switch from ON state to OFF State. Delay in SOI MOSFET is largest. This is main problem faced by SOI-MOSFET. DG-MOSFET shows comparable sub-threshold swing with MOSFET.

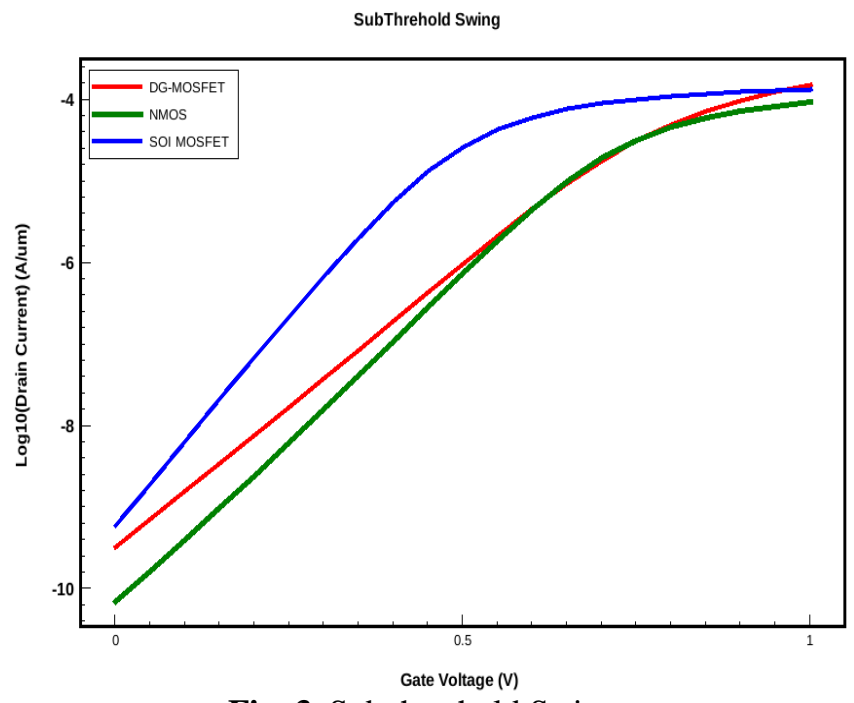

Fig. 3. Sub threshold Swing.

\subsection{DIBL}

Effective gate length reduces as Vds increases. Drain depletion region moves closer to source, resulting in significant field penetration from drain to source. Due to this penetration, potential barrier at source results in increase in drain current. This process called DIBL (Drain Induced Barrier Lowering)

$$
\mathrm{DIBL}=\frac{\delta V t}{\delta V d s}
$$

DIBL of NMOS is much larger which is expected to increase at lower gate length. Higher the DIBL higher the chance of device fails to go in off state. DIBL is least in DG-MOSFET, so it is more prominent candidate for further scaling.

DIBL

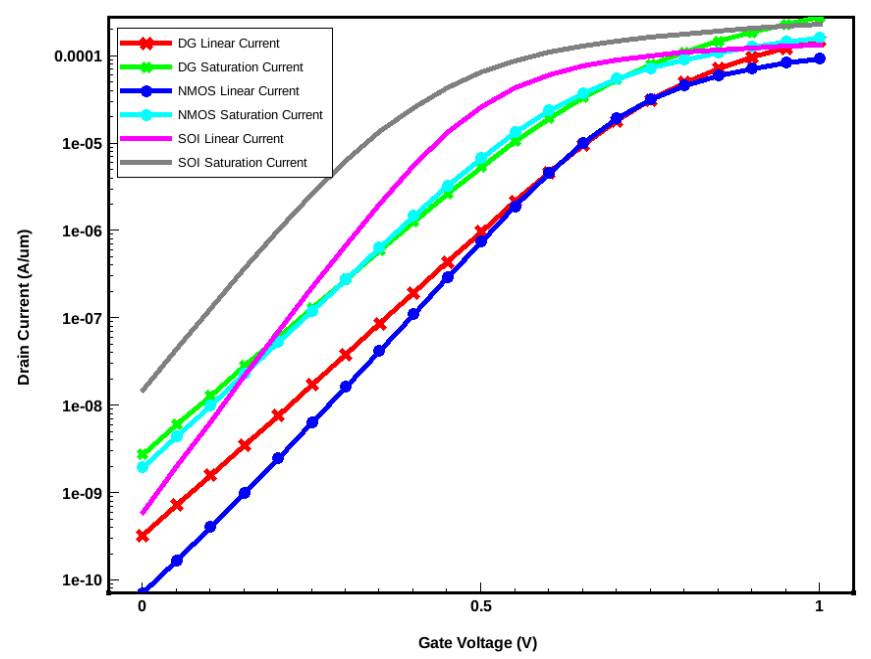

Fig. 4. DIBL

\subsection{Electrostatic Potential}

Electrostatic potential in lightly doped channel is obtained by solving 2D poisons equation:- 


$$
\frac{\partial^{2} \psi}{\partial x^{2}}+\frac{\partial^{2} \psi}{\partial y^{2}}+\frac{\partial^{2} \psi}{\partial z^{2}}=\frac{q N_{a}}{\epsilon s i}
$$

By solving above equation with appropriate boundary condition of device, we get potential inside channel region. Potential variation along length of devices is shown. It can be inferred from fig.5 that DG-MOSFET has greater channelControl compared to others. SOI-MOSFET and NMOS has relatively same control over channel.

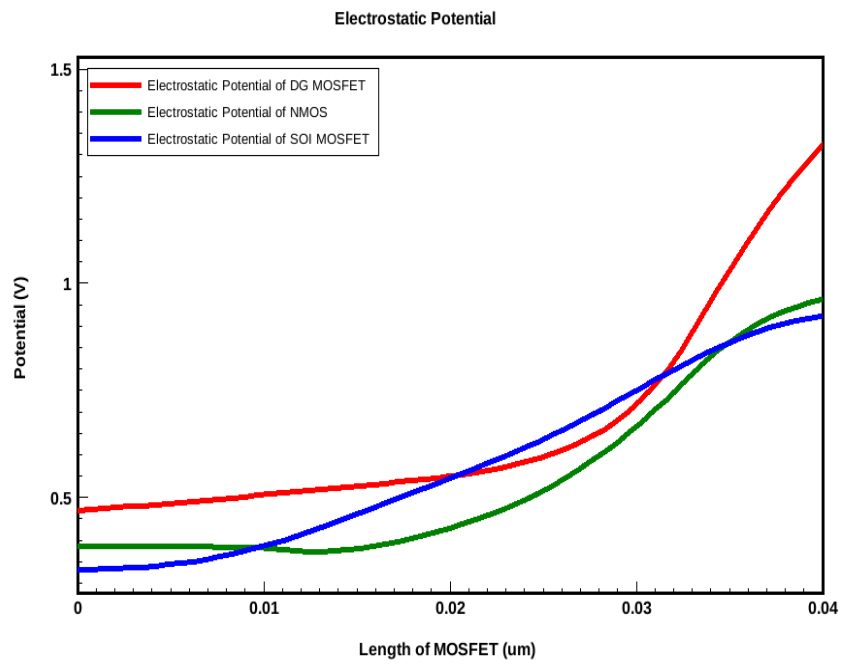

Fig. 5. Variation of Potential along Length of Device

\section{RESULT AND DISCUSSION}

All the structure are realized and plotted by using SENTAURUS TCAD. The comparison between different short channel effect are given below:- From Table II, DGMOSFET andNMOS has comparable sub threshold swing. It is better toHave higher sub threshold swing so that the device quicklyswitches from on to off state or vice-versa. In short the device is fasterIoff current in DG-MOSFET is least indicates less leakage

Table II- Device dimension and doping concentration

\begin{tabular}{|l|l|l|l|}
\hline Parameter & $\begin{array}{l}\text { DG- } \\
\text { MOSFET }\end{array}$ & $\begin{array}{l}\text { SOI- } \\
\text { MOSFET }\end{array}$ & NMOS \\
\hline $\begin{array}{l}\text { S.Swing } \\
(\mathrm{mV} / \mathrm{dec})\end{array}$ & 127.2 & 96.3 & 139.4 \\
\hline DIBL & 0.0422 & 0.133 & 0.166 \\
\hline Ion (A/um) & $1.312 \times 10^{-4}$ & $2.37 \times 10^{-4}$ & $1.6 \times 10^{-4}$ \\
\hline Ioff (A/um) & $1.03 \times 10^{-10}$ & $1.53 \times 10^{-9}$ & $1.976 \times 10^{-9}$ \\
\hline
\end{tabular}

Current. Ion current in all device is relatively same, thus Ion To Ioff ratio is maximum in DG-MOSFET.

DIBL of DG-MOSFET is 0.0422, which is comparatively veryless than other both device. DIBL of NMOS is highest signifiesgreater chance of device failed to min off condition. HigherDIBL least possibility of device scaling, so DGMOSFET ismore prominent candidate in planar technology for furtherscaling.

\section{CONCLUSION}

The simulation of SOI-NMOS, DG-MOSFET device has been studied. We found that DG-MOSFET has better controlover channel. It has comparable sub threshold swing and Ioncurrent to NMOS. In addition, least DIBL and Ioff current make itprominent candidate for further scaling.

\section{REFERENCES}

[1]. C.Hu, Modern Semiconductor Devices for Integrated Circuit, Prentice Hall, 2009.

[2]. C.H Wann et al, A Comaparative Study of Advanced MOSFET Concepts,,IEEE Trans. Electron Dev. 43(10) (1996)1742-1753.

[3]. T. Skotnicki et al , The End of CMOS Scaling,,IEEE Circ. Dev.Mag.21(1) (2005)16-26.

[4]. S.Veeraraghavan, et al,Short Channel Effect in SOI MOSFET, IEEETrans.Electron Dev.36(3) (1989) 522-528.

[5]. NarainArora, MOSFET Modeling for VLSI simulation, World Scientific,(287-305).

[6]. Xiaoping Liang, YuanTaur,A 2D Analitical Solution for SCE in DG-MOSFET, IEEE Trans. Electron Dev. 2004.

[7]. Susant Kumar Mohapatra et al ,Effect of Channel and Gate Engineer-ing on Double Gate MOSFET-A Comparitive Study, 978-1-4673-3136-4/12/31.00 (2012),IEEE.

[8]. N.BteAtan, et al,Effect of High K Dielctrics with Metal Gate forElectrical Characteristics of $18 \mathrm{~nm}$ NMOS Device, IEEE-ICSE (2014).

\section{BIOGRAPHIES}

Abhishek Kumar received the B.Tech. degrees in Electronics and Instrumentation from Galgotia's college of engineering and technology, Greater Noida, in 2013.He is currently pursuing the M.Tech. degree in VLSI and embedded system engineering at KIIT University, Bhubaneswar, Odisha. Her research interests include mainly focusing on device modeling.

Anil Kumar Swain received the diploma in Electronics and communication engineering from SCTE\&VT University Odisha India in 2010, B-Tech degree in electronics and communication engineering from BPUT University Odisha India in 2013 and Currently pursuing M-Tech in VLSI\&ES from KIIT University Odisha India. 